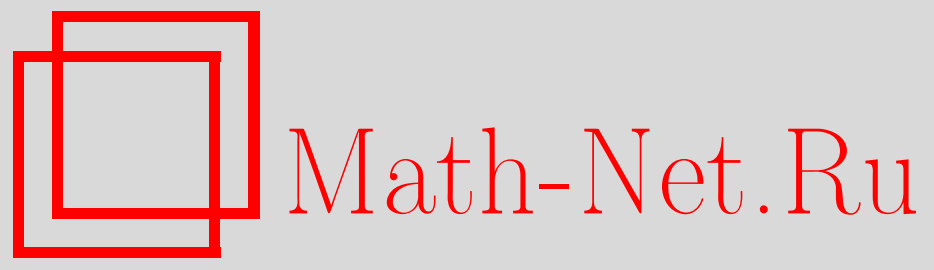

R. Rudzkis, M. Radavicius, Adaptive estimation of distribution density in the basis of algebraic polynomials, Теория вероятн. и ее примен., 2004, том 49, выпуск 1, 126-144

DOI: https://doi.org/10.4213/tvp239

Использование Общероссийского математического портала MathNet.Ru подразумевает, что вы прочитали и согласны с пользовательским соглашением

http://www.mathnet.ru/rus/agreement

Параметры загрузки:

IP : 54.224 .135 .184

26 апреля 2023 г., 16:24:49

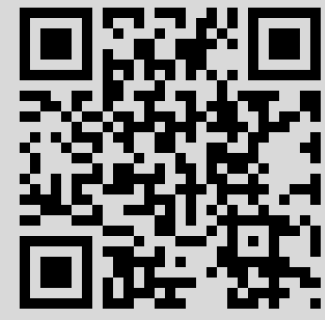




\section{ADAPTIVE ESTIMATION OF DISTRIBUTION DENSITY IN THE BASIS OF ALGEBRAIC POLYNOMIALS ${ }^{1)}$}

Статья посвящена задаче адаптивного статистического оценивания плотности распределения, определенной на конечном интервале. Рассматриваются оценки проективного типа, основанные на многочленах Якоби. Построена адаптивная статистическая оценка, являющаяся асимптотически минимаксной в смысле среднеквадратических потерь для всех множеств из семейства сжимающихся множеств функций различной гладкости. Условия гладкости формулируются в терминах $L_{2}$-норм остатков при аппроксимации плотностей распределений линейными комбинациями конечного числа первых многочленов Якоби. Обсуждается также обобщение этого результата на другие ортонормированные базисы, обладаюшие некоторыми естественными свойствами регулярности.

Ключевые слова и фразыл: адаптивное оценивание, локально минимаксное оценивание, многочлены Якоби, оценки проективного типа, среднеквадратические потери.

1. Introduction. In the statistical estimation of an unknown parameter (finite- or infinite-dimensional) one seeks to use optimal estimators. For this a mathematical definition of optimality is needed. In the Bayes setting this problem is solved, however, practical realization of this solution runs into another problem: to select a prior distribution of the parameter. In the recent decades the notion of minimax estimators has found increasing favour. From the viewpoint of applications, the main criticism is that their construction is guided by the worst (in the sense of identification accuracy) case of possible models. This results in a strong dependence of the estimating quality on a priori information. Significantly more flexible are adaptive estimators which have the same construction as minimax estimators, but they use more exact prior information specified on the basis of the sample. These estimators are far less sensitive to a priori information yet retaining the asymptotic minimax optimality.

Let us introduce necessary definitions. Let $\mathbf{X}=\left\{X_{1}, \ldots, X_{N}\right\}$ be a sample of independent and identically distributed (i.i.d.) random variables $X_{i}$

${ }^{*}$ Institute of Mathematics and Informatics, Akademijos 4, 2600 Vilnius, Lithuania; e-mail: rudzkis@ktl.mii.lt; mrad@ktl.mii.lt 
taking values in a compact convex set $K \subset \mathbf{R}^{d}$, and having a distribution function $F$. Let $f=d F / d \mu$ be a distribution density (d.d.) with respect to a finite measure $\mu$. For simplicity, we assume that $\mu(K)=1$.

Let $W$ be some set of d.d.'s and $\varrho(\psi, f)$ a loss function (pseudodistance). For a given statistical estimator $\hat{f}(\cdot)=\hat{f}(\cdot, \mathbf{X})$, denote $\delta(\hat{f}, f)=\mathbf{E} \varrho(\hat{f}, f)$, $\delta(\hat{f}, W)=\sup _{f \in W} \mathbf{E} \delta(\hat{f}, f)$. Then the minimax risk is defined by the equality

$$
\delta(W)=\inf _{\hat{f}} \delta(\hat{f}, W)
$$

where infimum is taken over all the estimators $\hat{f}$.

D e fin ition 1. An estimator $f^{*}(\cdot)=f^{*}(\cdot, \mathbf{X})$ is called an asymptotically minimax one (with respect to the pair $(\varrho, W)$ ) if and only if $\delta\left(f^{*}, W\right) \sim \delta(W)$.

Here and in the sequel the symbol $\sim$ designates the asymptotic equivalence of two sequences, i.e., $y_{N} \sim z_{N}$ means $\lim _{N \rightarrow \infty} y_{N} / z_{N}=1$. Here and everywhere below, unless otherwise stated, the asymptotics as $N \rightarrow \infty$ is considered.

Let $\left\{W_{\alpha}=W_{\alpha}(N), \alpha \in A\right\}$ be a given, dependent on $N$, family of neighborhoods of the distribution density.

D e f in ition 2. An estimator $f^{*}$ is called an adaptive estimator with respect to the family of neighborhoods $\left\{W_{\alpha}, \alpha \in A\right\}$ if

$$
\forall \alpha \in A \quad \delta\left(f^{*}, W_{\alpha}\right) \sim \delta\left(W_{\alpha}\right) .
$$

Usually the parameter $\alpha$ characterizes the «smoothness» of the set $W_{\alpha}$.

There is a wide bibliography devoted to the minimax and, especially, adaptive estimation. Let us give some relevant references. In the pioneering paper [31] M.S. Pinsker constructed the minimax estimator of a signal in noise for the squared $L_{2}$ distance $\varrho(f, g)=\|f-g\|_{2}^{2}$ and $W$ being an ellipsoid in $L_{2}$. This result was extended to other nonparametric models: distribution and spectral densities [13], [14] and regression function [21], [12] (see also [37]). A.P. Korostelev [26] have found the minimax estimator for the Hölder classes with $L_{\infty}$ risk (see also [5]). In the case where $W$ is a certain class of analytical functions, the minimax estimators for the $L_{p}$ risk were proposed in [20] $(p=\infty)$ and [23] $(1 \leqslant p<\infty)$. To the authors' knowledge, these three special cases exhaust the list of minimax estimating problems solved. So-called rate minimax estimators that have optimal rate of convergence of the minimax risk have been investigated far more extensively. See, for instance, [24], [4], [36], [2], [3], [9], [25], [10], [8], and references cited therein. The respective optimal rate adaptive estimators (we use the terminology of [38]) have been proposed in [28], [29], [30], [22], [6], [7] among others. Note that, for the pointwise risk on the scale of Hölder or $L_{2}$-Sobolev 
classes even optimal rate adaptive estimators do not exist: a logarithmic factor is a necessary payment for adaptation [38]. The nonasymptotic theory of adaptive estimation and a review of recent results on adaptivity are presented in [1]. The adaptive minimax estimators are obtained only in the case of estimating in $L_{2}$ [15], [21], [18] (see also [34], [11], [16]). As it is shown in [30], adaptive estimators can not achieve the asymptotic minimax risk for the sup-norm losses on the scale of Hölder classes (Korestelev's case).

The presented paper is closely related to [33]. Although [33] deals with a more general weighted mean-square risk, for simplicity, we discuss the main result only in a special case of usual $L_{2}$-losses $\varrho(\psi, f)=\|\psi-f\|_{2}^{2}$. Then it would be natural to define the neighborhoods $W_{\alpha}, \alpha \in A$, as contracting balls in $L_{2}$. However, since the estimating accuracy heavily depends upon the smoothness of the unknown d.d., the definition of neighborhoods under consideration should also include certain smoothness conditions.

Let $u=\left\{u_{k}\right\}_{0}^{\infty}$ be an orthonormal basis in the space $L_{2}=L_{2}(K, \mu)$ and $u_{0}(\cdot) \equiv 1$. Denote

$$
\begin{gathered}
W_{b}=\left\{f:\left\|f-(f)_{k-1}\right\|_{2}^{2} \leqslant b_{k}, k=1,2, \ldots ;\right. \\
\left.0 \leqslant f(x) \leqslant C_{1}, \int_{K} f d \mu=1\right\}, \\
W_{g, b}=W_{g, b}(N)=\left\{f: f \in W_{b},\|f-g\|_{2}^{2} \leqslant \varepsilon_{N}\right\} .
\end{gathered}
$$

Here $b=\left\{b_{k}\right\}_{1}^{\infty}$ is a given nonnegative sequence, $(f)_{k}$ signifies the orthogonal projection of the d.d. $f$ onto the linear subspace $\operatorname{span}\left\{u_{0}, \ldots, u_{k}\right\}$, and $\varepsilon_{N} \rightarrow 0$ as $N \rightarrow \infty$. In paper [33], under quite general conditions for the orthobasis $u$ and the set $\mathscr{B}$ of sequences $b$, it is established that for each $b \in \mathscr{B}$ there exists an estimator $f^{*}(\cdot)=f^{*}(\cdot, b, \mathbf{X})$ possessing the property of local asymptotic minimaxity:

$$
\forall g \in W_{b}^{\circ} \quad \delta\left(f^{*}, W_{g, b}\right) \sim \delta\left(W_{g, b}\right),
$$

where $W_{b}^{\circ}$ represents the «interior» of $W_{b}$,

$W_{b}^{\circ}=\left\{g: u_{0}+\lambda\left(g-u_{0}\right) \in W_{b}\right.$ for some $\lambda>1,\left\|g-(g)_{k}\right\|_{\infty} \rightarrow 0$ as $\left.k \rightarrow \infty\right\}$,

$\|\cdot\|_{\infty}$ is the supremum norm. Thus, the estimator $f^{*}$ achieves the asymptotic efficiency in both parametric (the sequence $b$ has only a finite number of nonzero terms) and nonparametric ( $b$ is strictly positive) cases. (Let us stress, however, that $f^{*}$ is not an adaptive estimator since it requires $a$ priori knowledge of the sequence $b$ which determines the smoothness of the d.d. f.) It seems likely to be the first estimator having this property. The estimator involves the statistical analysis of the Fisher information matrix and this feature distinguishes it from the traditionally used linear projective estimators (see [14], [11], [16], [18], [19], and others). 
Proceeding from [33], conceptually it is not difficult to build up an adaptive estimator $f^{*}(\cdot)=f^{*}(\cdot, \mathbf{X})$ possessing the same asymptotic efficiency without prior knowledge of the sequence $b$. However, the proof of this fact is rather complicate and cumbersome. Therefore we present here a simpler adaptive estimating procedure that fulfils (2) for strictly positive sequences $b$ but is not efficient in the parametric case. The procedure ignores subtleties of the structure of the Fisher information matrix since for the basis of Jacobi polynomials in the nonparametric case it can be replaced by a certain diagonal matrix without violating (2).

The extension of the results to more general orthonormal bases and to both the nonparametric and parametric cases will be presented in the next paper of the authors.

The rest of the paper is organized as follows. We conclude this section with some notation. The main results are formulated in the next section. The last section contains the proofs.

General notation. By $C$, with or without indices, we denote absolute positive constants not necessarily the same in different places; for instance, $C_{f}$ stands for any constant dependent on $f$ only. Further, $\mathbf{N}=\{1,2, \ldots\}$, $a \vee b=\max (a ; b), a \wedge b=\min (a ; b), x^{+}=x \vee 0, \underline{x}=1-x$, Int $(x)$ is the integer part of $x$. The indicator (characteristic function) of an event $A$ is denoted by $\mathbf{I}_{A}$. The expression $\alpha_{N} \asymp \beta_{N}$ indicates that the sequences $\alpha_{N}$ and $\beta_{N}$ are of the same order, i.e., $\lim \sup _{N \rightarrow \infty}\left(\left|\alpha_{N} / \beta_{N}\right|+\left|\beta_{N} / \alpha_{N}\right|\right)<\infty$. For any finite set $T,|T|$ is the number of elements of the set $T$.

2. Main results. We will write $b \in \mathscr{B}_{\nu}, \nu \geqslant 0,\left(\mathscr{B} \stackrel{\text { def }}{=} \mathscr{B}_{0}\right)$ if the following conditions are fulfilled:

1) $b_{k}>0$ for all $k \in \mathbf{N}$ ( $W_{b}$ is actually nonparametric);

2) $b_{k} \downarrow 0$ as $k \uparrow \infty$ and

$$
\lim _{n \rightarrow \infty} \sup _{k \in \mathbf{N}} \frac{b_{k n}}{b_{k}}=0
$$

3) the parameter $\nu$ governs the decreasing rate:

$$
b_{k}=o\left(k^{-2 \nu} \ln ^{-1} k\right), \quad k \rightarrow \infty .
$$

It is not difficult to check that (3) implies (4) for some $\nu=\nu(b)>0$. Indeed, in view of (3) $\sup _{k \in \mathbf{N}} b_{k s} / b_{k} \leqslant \frac{1}{2}$ for some $s \in \mathbf{N}$. This inequality and monotonicity of $b_{k}$ imply $b_{k} \leqslant 2 b_{1} k^{-h}$ with $h=\ln _{s} 2>0$.

For $b \in \mathscr{B}$, set

$$
M=M(b, N)=\max \left\{k: b_{k} \geqslant k N^{-1}\right\} .
$$

Then (see, e.g., [32], [33])

$$
\delta\left(W_{b}\right) \asymp M N^{-1}
$$


As already pointed out, in the paper our attention is focused on the case, where $u=\left\{u_{k}\right\}_{0}^{\infty}$ is the orthonormal basis of Jacobi polynomials. This means that $K=[-1,1], u_{k}(\cdot)$ is a $k$ th order polynomial, $k=0,1, \ldots$, and

$$
d \mu(x)=2^{-(\beta+\gamma+1)} B^{-1}(\beta+1, \gamma+1)(1-x)^{\beta}(1+x)^{\gamma} d x, \quad x \in[-1,1],
$$

where $B(\cdot, \cdot)$ is the Beta function, $\gamma>-1, \beta>-1$. Let us recall that special cases of Jacobi polynomials are Chebyshev $\left(\gamma=\beta=-\frac{1}{2}\right)$ and Legendre $(\gamma=\beta=0)$ polynomials.

Let $c_{k}=c_{k}(f), k \in \mathbf{N}$, denote the Fourier coefficients of a function $f \in W_{b}$ and let $\widehat{c}_{k}=N^{-1} \sum_{j=1}^{N} u_{k}\left(X_{j}\right), k \in \mathbf{N}$, be the empirical Fourier coefficients. Set

$$
a_{g}=\frac{1}{\pi} \int_{-1}^{1}\left(1-x^{2}\right)^{-1 / 2} g(x) d x .
$$

From [32] it is easy to derive the following statement.

Theorem 1. Suppose that $q \stackrel{\text { def }}{=} \max (\gamma, \beta)+\frac{1}{2} \geqslant 0$ and $b \in \mathscr{B}_{q}$. Let $\varepsilon_{N}=\varepsilon_{N}(b) \rightarrow 0$ and let $n=n(N, b)$ be chosen so that $M / N=o\left(\varepsilon_{N}\right)$, $b_{n}=o(M / N)$. Then the estimator

$$
f^{*}=u_{0}+\sum_{j=1}^{n} \frac{s_{j}^{*} \widehat{c}_{j}}{s_{j}^{*}+a_{g} / N} u_{j}
$$

is asymptotically minimax for all $W_{g, b}, g \in W_{b}^{\circ}$, and

$$
\delta\left(W_{g, b}\right) \sim \delta\left(f^{*}, W_{g, b}\right) \sim \Delta(g, b) \stackrel{\text { def }}{=} \frac{a_{g}}{N} \sum_{j=1}^{\infty} \frac{s_{j}^{*}}{s_{j}^{*}+a_{g} / N} .
$$

Here $\left\{s_{j}^{*}\right\}$ is a sequence maximizing all the sums $\sum_{j \geqslant k} s_{j}, k \in \mathbf{N}$, over the set of all nonincreasing nonnegative sequences $\left\{s_{j}\right\}_{1}^{\infty}$ satisfying $\sum_{j \geqslant k} s_{j} \leqslant b_{k}$ for all $k$.

The optimal estimator $f^{*}$ given in (7) is obtained in the following way. Let the set $\Omega$ consist of all nonnegative nonincreasing sequences $\omega=\left\{\omega_{k}\right\}_{1}^{\infty}$ with $\omega_{1} \leqslant 1$. Consider projective-type estimators

$$
\hat{f}=u_{0}+\sum_{j=1}^{n} \omega_{j} \widehat{c}_{j} u_{j}
$$

where $\omega \in \Omega$ are determinate (nonrandom) weight coefficients. Then the mean-square risk $\mathbf{E}\|\hat{f}-f\|^{2}$ of $\hat{f}$ for appropriately chosen (sufficiently large) $n$ is well approximated by the quantity

$$
\Psi_{a, n}(\omega, c)=\sum_{k=1}^{n}\left[\omega_{k}^{2} \frac{a}{N}+\left(1-\omega_{k}\right)^{2} c_{k}^{2}\right]
$$


with $a=a_{f}$. Since $a_{f} \sim a_{g}$ for $f \in W_{g, b}$, the optimal estimator $f^{*}$ among estimators (8) can be defined by the equality

$$
f^{*}=u_{0}+\sum_{j=1}^{n} \omega_{j}^{*} \widehat{c}_{j} u_{j}
$$

where

$$
\omega^{*}=\underset{\omega \in \Omega}{\arg \min } \max _{f \in W_{g, b}} \Psi_{a_{g}, n}(\omega, c) .
$$

It is not difficult to verify that $\omega_{j}^{*}=s_{j}^{*} /\left(s_{j}^{*}+a_{g} / N\right), j \in \mathbf{N}$, and

$$
\Delta(g, b) \sim \Psi_{a_{g}, n}\left(\omega^{*}, s^{*}\right) \asymp M N^{-1} .
$$

The following theorem is the main result of the paper.

Theorem 2. There exists an estimator $f^{*}$ (independent of $g$ and $b$ ) such that

$$
\delta\left(f^{*}, W_{g, b}\right) \leqslant \Delta(g, b)(1+o(1))
$$

for all $b \in \mathscr{B}$ and $g \in W_{b}^{\circ}$.

Corollary 1. Let the sequence $\varepsilon_{N}=\varepsilon_{N}(b)$ satisfy the relation $M / N=o\left(\varepsilon_{N}\right)$. Then there exists an adaptive estimator $f^{*}$ for the class of neighborhoods $\left\{W_{g, b}, b \in \mathscr{B}_{q}, g \in W_{b}^{\circ}\right\}$, where $q \geqslant 0$ is defined in Theorem 1 .

$\mathrm{R}$ e mark 1 . If $b \in \mathscr{B}_{\nu}$ where $\nu>q+\frac{1}{2}$, then $\left\|g-(g)_{k}\right\|_{\infty} \rightarrow 0$ as $k \rightarrow \infty$ for each $g \in W_{b}$ (see, e.g., [35]). Thus, this condition can be omitted in the definition of $W_{b}^{\circ}$ provided $\nu>q+\frac{1}{2}$.

Construction of the adaptive estimator. The construction of the adaptive estimator $f^{*}$ is based on formulas (9)-(11) with the following substitutions.

Let $\Pi_{i}, i=0,1,2,3$, be subsets of $\Pi=\{1, \ldots, N\}$ and $\Pi=\bigcup_{i=0}^{3} \Pi_{i}$. They define the four subsamples $\mathbf{X}\left(\Pi_{i}\right)=\left\{X_{t}, t \in \Pi_{i}\right\} \subset \mathbf{X}$ of the size $N_{i}=\left|\Pi_{i}\right|$.

1) A statistical estimator $\widehat{a}$ based on the subsample $\mathbf{X}\left(\Pi_{0}\right)$ is substituted for $a_{g}$.

2) Instead of the determinate number $n$ a certain statistics $n^{*}$ of the subsample $\mathbf{X}\left(\Pi_{1}\right)$ is used.

3) The optimal weights $\omega^{*}$ are replaced by adaptive ones (we retained for them the same notation) calculated on the basis of the subsample $\mathbf{X}\left(\Pi_{2}\right)$. The adaptive weights are obtained via minimization of the estimators of $\Psi_{a_{g}, n}(\omega, c)$ given by

$$
\widehat{\Psi}_{\widehat{a}, n^{*}, D}(\omega)=\sum_{k=1}^{n^{*}}\left[\omega_{k}^{2} \frac{\widehat{a}}{N}+\left(1-\omega_{k}\right)^{2} \widehat{c_{k}^{2}}(D)\right],
$$


where $D \subset \Pi_{2}$ and

$$
\widehat{c_{k}^{2}}(D)=\frac{1}{|D|(|D|-1)} \sum_{t, \tau \in D, t \neq \tau} u_{k}\left(X_{t}\right) u_{k}\left(X_{\tau}\right)
$$

is the unbiased estimator of $c_{k}^{2}, k \in \mathbf{N}$, based on the observations $\mathbf{X}(D)=$ $\left\{X_{i}, i \in D\right\}$.

4) The empirical Fourier coefficients $\widehat{c}_{k}$ are replaced by statistical estimators $c_{k}^{*}$, asymptotically equivalent to the former (in the sense of meansquare error). Actually, $c^{*}=\left\{c_{k}^{*}\right\}_{1}^{\infty}$ are the empirical Fourier coefficients based on the subsample $\mathbf{X}\left(\Pi_{3}\right)$. The asymptotic equivalence is ensured by the condition $N_{3} \sim N$.

It is natural to take $\Pi_{i} \equiv \Pi$. However, in this case, the proof of adaptivity of $f^{*}$ is very complicated in view of statistical dependence of the statistics $\widehat{a}, n^{*}, \omega^{*}, c^{*}$. Therefore, we take the disjoint sets $\Pi_{i}$, so $N_{0}+\cdots+N_{3}=N$.

Now let us give explicit description of the estimators involved. Fix $\alpha \in\left(\frac{1}{2}, 1\right)$ and set $N_{0}=\operatorname{Int}\left(N^{\alpha}\right)$.

In view of $(6)$

$$
a_{f}=\int_{-1}^{1} \psi(x) d F(x)
$$

where

$$
\psi(x)=\frac{1}{\pi \sqrt{1-x^{2}}}\left(\frac{d \mu}{d x}(x)\right)^{-1}
$$

Define

$$
\widehat{a}=\frac{1}{N_{0}} \sum_{t \in \Pi_{0}} \psi_{N}\left(X_{t}\right)
$$

with $\psi_{N}(x)=\psi(x) \wedge \sqrt{N}$. For estimation of $n^{*}$, we take a sequence of index sets $D_{i}$ such that $D_{s} \subset \cdots \subset D_{2} \subset D_{1} \subset \Pi \backslash \Pi_{0},\left|D_{k}\right|=\operatorname{Int}(N /(4 k))$, and $s=\operatorname{Int}\left(\ln _{2} N\right)$. For a fixed $\beta \in\left(\frac{1}{2}, 1\right)$, define

$$
k^{*}=1 \vee \max \left\{k: k \in\{1, \ldots, s\}, \quad \sum_{i=2^{k-1}}^{2^{k}} \widehat{c_{i}^{2}}\left(D_{k}\right)>\frac{2^{k \beta}}{N}\right\}
$$

and $n^{*}=2^{k^{*}}, \Pi_{1}=D_{k^{*}}$.

Set $N_{2}=2 N_{1}$ and split the set $\Pi_{2}$ into two nonintersecting subsets of equal cardinalities, $\Pi_{2}=D \cup \widetilde{D}$, and set $\omega_{(\cdot)}^{*}=\widehat{\omega}_{(\cdot)} \vee \widetilde{\omega}_{(\cdot)}$, where $\widehat{\omega}=$ $\omega\left(\widehat{\Psi}_{\widehat{a}, n^{*}, D}\right), \widetilde{\omega}=\omega\left(\widehat{\Psi}_{\widehat{a}, n^{*}, \widetilde{D}}\right)$, and

$$
\omega\left(\Psi_{(\cdot)}\right)=\underset{\omega \in \Omega}{\arg \min } \Psi_{(\cdot)}(\omega) .
$$

Finally, we take

$$
c_{j}^{*}=N_{3}^{-1} \sum_{t \in \Pi_{3}} u_{j}\left(X_{t}\right), \quad j \in \mathbf{N} .
$$


Generalization. Now we will describe the extensions of the results to more general orthonormal bases in $L_{2}(K, \mu)$ possessing some additional regularity properties.

(U0) The functions $u_{k}, k=0,1,2, \ldots$, are differentiable with the bounded gradients $\nabla u_{k}$.

(U1) There exist a function $\psi \in L_{p}, p>1$, and $\tau>0$ such that, for each $g \in W_{b}, b \in \mathscr{B}$,

$$
a_{g} \stackrel{\text { def }}{=} \lim _{k \rightarrow \infty} \int_{K} u_{k}^{2}(x) g(x) \mu(d x)=\int_{K} \psi(x) g(x) \mu(d x) \geqslant \tau
$$

and the convergence in (17) is uniform with respect to $g \in W_{b}$.

(U2) There exists a sequence $\left\{m_{k}\right\} \subset \mathbf{N}, m_{k}=o(\sqrt{k})$, such that for each $l \in \mathbf{N}$,

$$
\sum_{i=0}^{k} \sum_{j>i+m_{k}}\left(\int_{K} u_{l}(x) u_{i}(x) u_{j}(x) \mu(d x)\right)^{2}=o(k), \quad k \rightarrow \infty .
$$

We write $b \in \mathscr{B}_{+}$if $b \in \mathscr{B}$ and

$$
\frac{1}{N}\left\|\sum_{k=1}^{M} u_{k}^{2}(\cdot)\right\|_{\infty} \ln \left(2+\frac{1}{N}\left\|\sum_{j=1}^{M}\left|\nabla u_{j}\right|^{2}\right\|_{\infty}\right) \longrightarrow 0, \quad N \rightarrow \infty,
$$

where $M=M(b, N)$ is introduced in (5).

Theorem 3. The statements of Theorems 1, 2 and Corollary 1 are valid for any orthonormal basis u satisfying conditions (U0)-(U2) provided the set $\mathscr{B}_{q}$ of sequences $b$ in Theorem 1 and Corollary 1 is replaced by $\mathscr{B}_{+}$.

\section{Proof.}

3.1. Auxiliary results. Let $v_{j}(m)$ denote the conditional variance of $c_{j}^{*}$ under the condition $N_{3}=m$.

Lemma 1. Let $f \in W_{b}, b \in \mathscr{B}$, and let $a_{f}$ be defined by (6). Then $v_{j}(m) \leqslant\|f\|_{\infty} / N_{3}$ and

$$
\lim _{j \rightarrow \infty} \lim _{m \rightarrow \infty} m v_{j}(m)=a_{f} .
$$

The convergence in (19) is uniform with respect to $f \in W_{b}$.

$\mathrm{Pr}$ o of. The proof follows directly from the equality

$$
m v_{j}(m)=\int_{K} u_{j}^{2}(x) d F(x)-c_{j}^{2}
$$

and the fact that, for Jacobi polynomials, the limit functional $a_{g}$ in (17) takes the form (6) (see [32, Lemma 6, p. 32]).

Further we need additional notation. $\lambda(x)$ denotes any function of the type $\lambda(x)=C \ln ^{2}\left(x^{+}+2\right) /\left(x^{+}+2\right)$. A centered random variable $\xi$ is denoted by $\widehat{\xi}$, i.e., $\widehat{\xi}=\xi-\mathbf{E} \xi$. 
For any $T \subset \mathbf{R}^{1}$ and any sequence $Y=\left\{Y_{t}\right\}_{1}^{\infty}$, let $S_{T}(Y)=\sum_{t \in T \cap \mathbf{N}} Y_{t}$ and, assuming that $T$ is bounded, $\bar{Y}_{T}=S_{T}(Y) /|T \cap \mathbf{N}|$. Put

$$
\Gamma_{T}=S_{T}\left(c^{2}\right), \quad \gamma_{T}=\Gamma_{T}|T|^{-1} .
$$

Thus $\gamma_{[a, b]}$ stands for $\gamma_{T}$ with $T=[a, b]$. For $n, k \in \mathbf{N}$, define

$$
\gamma_{k}=\gamma_{k}(n)= \begin{cases}\gamma_{[p, q]}, & \text { if } \quad k \leqslant n, \\ 0, & \text { if } k>n\end{cases}
$$

here the integers $p=p(k, n)$ and $q=q(k, n)$ are defined by the relations

$$
1 \leqslant p \leqslant k \leqslant q \leqslant n: \gamma_{[p, q]}=\min _{1 \leqslant i \leqslant k} \gamma_{[i, q]}=\max _{n \geqslant j \geqslant k} \gamma_{[p, j]} .
$$

Let $D \subset \Pi$. The quantities $\widehat{\Gamma_{T}}(D), \widehat{\gamma_{T}}(D), \widehat{\gamma_{k}}(n, D), \widehat{p}(k, n)$ and $\widehat{q}(k, n)$ are defined by the same equations (20)-(22) but with $\left\{c_{k}^{2}\right\}$ replaced by $\left\{\widehat{c}_{k}^{2}(D)\right\}$. Also set $\widehat{\gamma}_{k}=\widehat{\gamma}_{k}(n, D) \stackrel{\text { def }}{=} \gamma_{k}-\widehat{\gamma}_{k}(n, D), k \in \mathbf{N}$.

A bit different definition of $\Psi_{a, n}(\omega)$ is more convenient in the proof. Redefine (9) as follows

$$
\Psi_{a, n}(\omega)=\sum_{k=1}^{n}\left[\omega_{k}^{2} \frac{a}{N}+\left(1-\omega_{k}\right)^{2} c_{k}^{2}\right]+\sum_{k>n} c_{k}^{2} .
$$

It easy to verify, using this expression, (12) and (16), that, for any $a>0$ and $n \in \mathbf{N}$,

$$
\omega_{k}\left(\Psi_{a, n}\right)=\frac{\gamma_{k}(n)}{\gamma_{k}(n)+a N^{-1}} \quad \text { and } \quad \omega_{k}\left(\widehat{\Psi}_{\widehat{a}, \widehat{n}, D}\right)=\frac{\widehat{\gamma}_{k}^{+}(\widehat{n}, D)}{\widehat{\gamma}_{k}^{+}(\widehat{n}, D)+\widehat{a} N^{-1}} .
$$

By the definition of $\left\{\widehat{c_{k}^{2}}\right\}$ in (13), we have $\widehat{c_{k}^{2}}(D)=c_{k}^{2}+2 \xi_{k}(D)+\zeta_{k}(D)$ where

$$
\begin{aligned}
& \xi_{k}(D)=\frac{c_{k}}{|D|} \sum_{t \in D} \widehat{u}_{k}\left(X_{t}\right), \\
& \zeta_{k}(D)=\frac{1}{|D|(|D|-1)} \sum_{t, \tau \in D, t \neq \tau} \widehat{u}_{k}\left(X_{t}\right) \widehat{u}_{k}\left(X_{\tau}\right),
\end{aligned}
$$

and $\widehat{u}_{k}\left(X_{t}\right)=u_{k}\left(X_{t}\right)-c_{k}$.

Lemma 2. For any $T \subset \mathbf{N}$ and $D \subset \Pi$,

$$
\mathrm{D} S_{T}(\xi(D)) \leqslant \frac{C\|f\|_{\infty} \Gamma_{T}}{|D|}, \quad \mathrm{D} S_{T}(\zeta(D)) \leqslant \frac{C\|f\|_{\infty}^{2}|T|}{|D|^{2}} .
$$

P r o of. Put $X=X_{1}$. Then by definition (25)

$$
\begin{aligned}
\mathbf{D} S_{T}(\zeta) & =\frac{2}{|D|(|D|-1)} \sum_{i, j \in T}\left[\mathbf{E} \widehat{u}_{i}(X) \widehat{u}_{j}(X)\right]^{2} \\
& \leqslant \frac{2}{|D|(|D|-1)} \sum_{i, j \in T}\left(\int u_{i} u_{j} d F\right)^{2} \\
& \leqslant \frac{C|T|}{|D|^{2}} \max _{j \in T}\left\{\left\|u_{j} f\right\|_{2}^{2}\right\} \leqslant \frac{C|T|\|f\|_{\infty}^{2}}{|D|^{2}} .
\end{aligned}
$$


Further, by (24)

$$
|D| \mathbf{D} S_{T}(\xi)=\sum_{i, j \in T} c_{i} c_{j} \mathbf{E} \widehat{u}_{i}(X) \widehat{u}_{j}(X) \leqslant \int\left(\sum_{i \in T} c_{i} u_{i}\right)^{2} d F \leqslant C\|f\|_{\infty} \cdot \Gamma_{T} .
$$

Proposition 1. Suppose that for a nonnegative sequence $\beta=\left\{\beta_{k}\right\}_{1}^{\infty}$ and a sequence $Y=\left\{Y_{k}\right\}_{1}^{\infty}$ of random variables the inequalities

$$
\mathbf{E} S_{T}^{2}(Y) \leqslant S_{T}(\beta), \quad T \subset \mathbf{N}
$$

are fulfilled. Then

$$
\forall l \in \mathbf{N} \quad \mathbf{E} \max _{j=1, \ldots, l} S_{[1, j]}^{2}(Y) \leqslant C \ln ^{2}(1+l) S_{[1, l]}(\beta) .
$$

P r o o f. The statement is obvious for $l=2$. Now, suppose that it holds for some $l \geqslant 2$. Then

$$
\mathrm{E} \max _{j=1, \ldots, l} S_{(k, k+j]}^{2}(Y) \leqslant C_{0} \ln ^{2} l \cdot S_{(k, k+l]}(\beta) \quad \forall k=0,1,2, \ldots .
$$

We show that (27) implies

$$
\mathbf{E} \max _{j=1, \ldots, r} S_{[1, j]}^{2}(Y) \leqslant C_{0} \ln ^{2} r \cdot S_{[1, r]}(\beta)
$$

for $r=2 l$ provided $C_{0} \geqslant 1 / \ln ^{2} 2$. In fact, from the Cauchy-Schwartz inequality and the elementary inequality $2 x y \leqslant x^{2}+y^{2}$, it follows that

$$
\begin{aligned}
\mathbf{E} \max _{j \leqslant r} S_{[1, j]}^{2}(Y) \leqslant & \mathbf{E} \max _{j \leqslant l} S_{[1, j]}^{2}(Y)+\mathbf{E} \max _{l<j \leqslant r}\left(S_{[1, l]}(Y)+S_{(l, j]}(Y)\right)^{2} \\
\leqslant & \left(C_{0} \ln ^{2} l+1\right) S_{[1, l]}(\beta)+2 \ln l \sqrt{C_{0} S_{[1, l]}(\beta) S_{(l, r]}(\beta)} \\
& +C_{0} \ln ^{2} l \cdot S_{(l, r]}(\beta) \leqslant\left(C_{0} \ln ^{2} l+\sqrt{C_{0}} \ln l+1\right) S_{[1, r]}(\beta) \\
\leqslant & C_{0} \ln ^{2} r \cdot S_{[1, r]}(\beta) .
\end{aligned}
$$

Proposition 2. If (26) holds, then

$$
\mathbf{E} \sup _{i \in J, i \geqslant k+l} \bar{Y}_{[k, i]}^{2} \leqslant \lambda(l) \cdot \sup _{i \in J, i \geqslant k} \bar{\beta}_{[k, i]}
$$

for any $J \subset \mathbf{N}, k \in \mathbf{N}$, and $l \geqslant 0$.

P r o o f. Let $l \geqslant 1, J_{r}=\left[k+2^{r-1} l, k+2^{r} l\right) \cap J$, and $H=\max _{i \in J, i \geqslant k} \bar{\beta}_{[k, i]}$. In view of Proposition 1 we obtain

$$
\begin{aligned}
\mathbf{E} \max _{j \in J, j \geqslant k+l} \bar{Y}_{[k, j]}^{2} & \leqslant \mathbf{E} \sum_{r=1}^{\infty} \frac{4^{1-r}}{l^{2}} \max _{j \in J_{r}} S_{[k, j]}^{2}(Y) \\
& \leqslant C \sum_{r=1}^{\infty} \frac{4^{-r}}{l^{2}} 2^{r} l \ln ^{2}\left(2^{r} l\right) H \leqslant \frac{C H \ln ^{2} l}{l} .
\end{aligned}
$$


Corollary 2. For any $k<r, k, r \in \mathbf{N}$, and $D \subset \Pi$,

$\mathbf{E} \max _{k \leqslant j \leqslant r} S_{[k, j]}^{2}(\xi(D)) \vee S_{[j, r]}^{2}(\xi(D)) \leqslant C_{f} \ln ^{2}(1+r-k) \Gamma_{[k, r]}|D|^{-1}$ and

$\mathrm{E} \max _{k \leqslant j \leqslant r} S_{[k, j]}^{2}(\zeta(D)) \vee S_{[j, r]}^{2}(\zeta(D)) \leqslant C_{f} \ln ^{2}(1+r-k) \cdot(r-k)|D|^{-2}$.

Corollary 3. Let $\widehat{\gamma}_{(\cdot)}=\gamma_{(\cdot)}-\widehat{\gamma}_{(\cdot)}(D)$, and $T \subset \mathbf{N}, r \in \mathbf{N}, l \geqslant 0$. Then

$\mathbf{E}_{j \in T} \widehat{\gamma}_{[j, r]}^{2} \leqslant C_{f} \lambda(l)\left(\frac{\max _{j \in T} \gamma_{[j, r]}}{|D|}+\frac{1}{|D|^{2}}\right), \quad$ if $\quad T \subset[1, r-l]$, and

$$
\mathbf{E} \sup _{j \in T} \widehat{\gamma}_{[r, j]}^{2} \leqslant C_{f} \lambda(l)\left(\frac{\max _{j \in T} \gamma_{[r, j]}}{|D|}+\frac{1}{|D|^{2}}\right), \quad \text { if } \quad T \subset[r+l, \infty) .
$$

Corollary 2 follows from Lemma 2 and Proposition 1. Corollary 3 is a consequence of Lemma 2 and Proposition 2. Given $a>0, n \in \mathbf{N}$, and $D \subset \Pi$, set $N_{1}=|D|, \widehat{\omega}=\omega\left(\widehat{\Psi}_{a, n, D}\right)$.

Lemma 3. Let $\omega=\omega\left(\Psi_{a, n}\right)$. For each $s, r \in \mathbf{N}, s \leqslant r \leqslant n$, such that $s=p(s, n), r=q(r, n)$, and $\Gamma_{[s, r]} \leqslant C r \gamma_{r}$, the following inequality holds:

$$
\mathbf{E} \sum_{k=s}^{r}\left(\omega_{r}-\widehat{\omega}_{k}\right)_{+}^{2} c_{k}^{2} \leqslant C_{f} \gamma_{s} \ln ^{4}(1+r) \cdot \frac{\gamma_{r} / N_{1}+N_{1}^{-2}}{\left(\gamma_{r}+a / N\right)^{2}} .
$$

P r o o f. Let $\widehat{\gamma}_{(\cdot)}=\widehat{\gamma}_{(\cdot)}(D)$. For a fixed $k \in \mathbf{N}$, set $\eta=\left(\gamma_{r}-\right.$ $\left.\min _{i \leqslant k} \widehat{\gamma}_{[i, r]}\right)_{+}$. For some $p, q \in \mathbf{N}$ we have $p \leqslant k \leqslant q$ and $\widehat{\gamma}_{k}=\widehat{\gamma}_{[p, q]}=$ $\max _{n \geqslant j \geqslant k} \widehat{\gamma}_{[p, j]} \geqslant \widehat{\gamma}_{[p, r]} \geqslant \min _{i \leqslant k} \widehat{\gamma}_{[i, r]}$, and hence $\left(\omega_{r}-\widehat{\omega}_{k}\right)_{+} \leqslant\left(\gamma_{r}-\widehat{\gamma}_{k}^{+}\right)_{+} /\left(\gamma_{r}+\right.$ $a / N) \leqslant \eta /\left(\gamma_{r}+a / N\right)$. Consequently,

$$
\mathbf{E}\left(\omega_{r}-\widehat{\omega}_{k}\right)_{+}^{2} \leqslant \frac{\mathbf{E} \eta^{2}}{\left(\gamma_{r}+a N^{-1}\right)^{2}} .
$$

Denote

$$
\begin{aligned}
I_{j} & =\left\{i: 1 \leqslant i \leqslant k, 2^{j} \leqslant \gamma_{[i, r]} \gamma_{r}^{-1}<2^{j+1}\right\}, \\
l_{j} & =1+r-\max \left\{i: i \in I_{j}\right\} .
\end{aligned}
$$

By the definitions of $I_{j}, l_{j}$, and $\Gamma_{[k, r]}($ see $(20))$,

$$
l_{j} \geqslant \frac{\Gamma_{[k, r]}}{\gamma_{r} 2^{j+1}} .
$$

Since $\min _{i \leqslant r} \gamma_{[i, r]} \leqslant \gamma_{r}$, we have $\eta=\max _{j \geqslant 0} \eta_{j}$, where $\eta_{j}=\left(\gamma_{r}-\right.$ $\left.\min _{i \in I_{j}} \widehat{\gamma}_{[i, r]}\right)_{+}$. Thus,

$$
\mathbf{E} \eta^{2} \leqslant \sum_{j=0}^{\infty} \mathbf{E} \eta_{j}^{2}
$$


In view of Corollary 3 and the definitions of $I_{j}$ and $\hat{\gamma}$, we can write

$$
\mathbf{E} \eta_{0}^{2} \leqslant \mathbf{E} \max _{i \in I_{0}} \widehat{\gamma}_{[i, r]}^{2} \leqslant C_{f} \lambda\left(l_{0}\right)\left(\gamma_{r} N_{1}^{-1}+N_{1}^{-2}\right)
$$

and, applying in addition the Chebyshev inequality,

$$
\begin{aligned}
\mathbf{E} \eta_{j}^{2} & \leqslant \gamma_{r}^{2} \mathbf{P}\left\{\eta_{j}>0\right\} \leqslant \gamma_{r}^{2} \mathbf{P}\left\{-\min _{i \in I_{j}} \widehat{\gamma}_{[i, r]}>\left(2^{j}-1\right) \gamma_{r}\right\} \\
& \leqslant C_{f} \lambda\left(l_{j}\right) 4^{-j}\left(2^{j} \gamma_{r} N_{1}^{-1}+N_{1}^{-2}\right) .
\end{aligned}
$$

Since $\Gamma_{[k, r]} \leqslant C r \gamma_{r}$, the last inequality and formulas (29)-(34) yield

$$
\mathbf{E}\left(\omega_{r}-\widehat{\omega}_{k}\right)_{+}^{2} \leqslant C_{f} \ln ^{3}(1+r) \cdot \frac{\gamma_{r}^{2} N_{1}^{-1}+\gamma_{r} N_{1}^{-2}}{\Gamma_{[k, r]}\left(\gamma_{r}+a N^{-1}\right)^{2}} .
$$

Further,

$$
\sum_{k=s}^{r} \frac{c_{k}^{2}}{\Gamma_{[k, r]}} \leqslant C \ln (1+r) \frac{\gamma_{s}}{\gamma_{r}} .
$$

It is not difficult to verify this inequality taking into account the condition $s=p(s, r), r=q(r, n)$. Inequalities (36) and (37) imply (28).

Corollary 4. For each $k \in \mathbf{N}$, we have $\mathbf{E} \widehat{\gamma}_{k}^{2} \leqslant C_{f}\left(\gamma_{k} /|D|+|D|^{-2}\right)$.

P r o o f. By making use of the definitions of $\gamma_{(\cdot)}, \widehat{\gamma}_{(\cdot)}, p$, and $q$ (see (21), and (22)), we conclude that

$$
\Delta_{1} \stackrel{\text { def }}{=} \min _{i \leqslant q} \widehat{\gamma}_{[i, q]} \leqslant \widehat{\gamma}_{k} \leqslant \max _{j \geqslant p} \widehat{\gamma}_{[p, j]} \stackrel{\text { def }}{=} \Delta_{2} .
$$

The required inequality for $\mathbf{E} \Delta_{1}^{2}$ follows from (33)-(35) and for $\mathbf{E} \Delta_{2}^{2}$ from Corollary 3.

Let the notation introduced before Lemma 3 hold, $\widetilde{n}=n \vee M$, and let $\widetilde{D} \subset \Pi$ be an arbitrary set satisfying $|\widetilde{D}|=|D|=N_{1}, \widetilde{D} \cap D=\varnothing$. Set $\widehat{\hat{\omega}}_{(\cdot)}=\widehat{\omega}_{(\cdot)} \vee \omega_{(\cdot)}\left(\Psi_{a, n, \widetilde{D}}\right)$.

Lemma 4. For any $\tau \in\left(0, \frac{1}{2}\right]$,

$$
\mathbf{E} \Psi_{a, n}(\widehat{\widehat{\omega}}) \leqslant \Psi\left[\left(1+\sqrt{\frac{C_{f} \ln ^{5} \tilde{n}}{\tau M}} \cdot \frac{N}{N_{1}}\right)^{2}+C \tau+\frac{C_{f} N^{2}}{\widetilde{n} N_{1}^{2}}\right],
$$

where

$$
\Psi \stackrel{\text { def }}{=} \min _{\omega \in \Omega} \Psi_{a, n}(\omega) .
$$

P r o o f. Let $\omega=\omega\left(\Psi_{a, n}\right)$. First we estimate $\mathbf{E} \Delta$, where

$$
\Delta=\sum_{j=1}^{n}\left(\underline{\widehat{\hat{\omega}}}_{j}^{2}-\underline{\omega}_{j}^{2}\right) c_{j}^{2}
$$


(Recall that $\underline{\omega}_{j}=1-\omega_{j}$. ) To this end, define a decreasing sequence of natural numbers $r_{k}, k \in \mathbf{N}$, by the equalities $r_{0}=n, r_{k}=\max \left\{j: \underline{\omega}_{j}<(1-\tau) \underline{\omega}_{r_{k-1}}\right\}$. Next, put $h=\max \left\{k: \underline{\omega}_{r_{k}} \geqslant \widetilde{n}^{-2} \vee \underline{\omega}_{1}\right\}, s_{k}=r_{k+1}+1$.

By the definition, for any $k=0, \ldots, h$,

$$
\begin{gathered}
p\left(s_{k}, n\right)=s_{k}, \quad q\left(r_{k}, n\right)=r_{k}, \\
(1-\tau)^{k} \geqslant \underline{\omega}_{r_{k}} \geqslant \underline{\omega}_{s_{k}} \geqslant(1-\tau) \underline{\omega}_{r_{k}} .
\end{gathered}
$$

For $s_{k} \leqslant j \leqslant r_{k}$ denoting $\nu_{j}=\nu_{j}(k)=\left(\underline{\widehat{\hat{\omega}}}_{j}-\underline{\omega}_{r_{k}}\right)_{+}$and applying the inequality $\underline{\omega}_{r_{k}} \leqslant 2 \underline{\omega}_{j}$, we obtain

$$
\Delta \leqslant \sum_{j<s_{h}} \widehat{\widehat{\hat{\omega}}}_{j}^{2} c_{j}^{2}+\sum_{k=1}^{h} \sum_{j=s_{k}}^{r_{k}}\left[\left(\underline{\omega}_{r_{k}}^{2}-\underline{\omega}_{j}^{2}\right)+4 \nu_{j} \underline{\omega}_{j}+\nu_{j}^{2}\right] c_{j}^{2} \stackrel{\text { def }}{=} \Delta_{1}+\cdots+\Delta_{4} .
$$

When estimating $\mathbf{E} \Delta_{i}, i=1, \ldots, 4$, we frequently use the following obvious relations:

$$
\sum_{j=1}^{n} \underline{\omega}_{j} \gamma_{j}=\sum_{j=1}^{n} \frac{\omega_{j} a}{N} \leqslant \Psi, \quad C \Psi \geqslant \frac{M}{N}
$$

We have

$$
\mathbf{E} \Delta_{1} \leqslant C \sum_{j<s_{h}}\left[\underline{\omega}_{j}^{2}+\mathbf{P}^{2}\left\{\widehat{\gamma}_{j}<\frac{\gamma_{j}}{2}\right\}\right] c_{j}^{2} .
$$

By virtue of Corollary 4 and the Chebyshev inequality, $\mathbf{P}\left\{\widehat{\gamma}_{j}<\gamma_{j} / 2\right\} \leqslant$ $C_{f} /\left(N_{1} \gamma_{j}\right)$. Thus, from (43), (44), and the inequalities $\underline{\omega}_{j} \leqslant \tilde{n}^{-2}, \gamma_{j} \geqslant$ $\left(\widetilde{n}^{2}-1\right) a / N$, where $j<s_{h}$, we conclude that

$$
\mathrm{E} \Delta_{1} \leqslant C_{f}\left(\frac{N}{N_{1}}\right)^{2} \frac{\Psi}{\widetilde{n}}
$$

Further, (41) and (43) imply

$$
\Delta_{2} \leqslant C \tau \sum_{j=1}^{n} \omega_{j}^{2} c_{j}^{2} \leqslant C \tau \Psi .
$$

Application of the Cauchy-Schwartz inequality yields

$$
\mathbf{E} \Delta_{3} \leqslant 4\left(\sum_{j=s_{h}}^{n} \mathbf{E} \nu_{j}^{2} c_{j}^{2}\right)^{1 / 2}\left(\sum_{j=1}^{n} \mathbf{E} \underline{\omega}_{j}^{2} c_{j}^{2}\right)^{1 / 2} \leqslant C\left(\mathbf{E} \Delta_{4}\right)^{1 / 2} \Psi^{1 / 2} .
$$

It remains to estimate the last term $\mathbf{E} \Delta_{4}$. Since $\gamma_{j}=\gamma_{\left[s_{k}, r_{k}\right]}$ for $s_{k} \leqslant j \leqslant r_{k}$, from Lemma 3 and (41) we derive that

$$
\mathrm{E} \sum_{j=s_{k}}^{r_{k}} \nu_{j}^{2} c_{j}^{2} \leqslant C_{f} \ln ^{4} \tilde{n} \cdot \frac{N}{N_{1}^{2}} \leqslant \frac{C_{f} \ln ^{4} \tilde{n}}{M}\left(\frac{N}{N_{1}}\right)^{2} \Psi .
$$


Because $h \leqslant \ln \tilde{n} / \tau$, from (42) and (45)-(48) it follows that

$$
\mathbf{E} \Delta \leqslant\left(\frac{C_{f} N^{2}}{\widetilde{n} N_{1}^{2}}+C \tau+\max _{\alpha=1,1 / 2}\left\{\left[\frac{c_{f} \ln ^{5} \tilde{n}}{\tau M}\left(\frac{N}{N_{1}}\right)^{2}\right]^{\alpha}\right\}\right) \Psi .
$$

Now let

$$
\Delta=\frac{a}{N} \sum_{j=1}^{n}\left(\widehat{\omega}_{j}^{2}-\omega_{j}^{2}\right)
$$

The expectation of (50) is estimated in a similar way as that of (39). The natural numbers $s_{j}$ and $r_{j}$ are defined sequentially by equalities $s_{0}=1$, $s_{k}=\min \left\{j: \omega_{j}<(1-\tau) \omega_{s_{k-1}}\right\}, h=\min \left\{k: \omega_{s_{k}} \leqslant \tilde{n}^{-2} \vee \omega_{n}\right\}, r_{h}=n$, $r_{k}=s_{k+1}-1$ for $k<h$. It is clear that (40) holds for $k=0, \ldots, h$, and

$$
(1-\tau)^{k} \geqslant \omega_{s_{k}} \geqslant \omega_{r_{k}}(1-\tau)^{-1}, \quad \text { if } k<h .
$$

Set $\nu_{j}=\nu_{j}(k)=\left(\widehat{\omega}_{j}-\omega_{s_{k}}\right)_{+}$for $s_{k} \leqslant j \leqslant r_{k}$. Then

$$
\Delta=\frac{a}{N} \sum_{k=1}^{h} \sum_{j=s_{k}}^{r_{k}}\left[\left(\omega_{s_{k}}^{2}-\omega_{j}^{2}\right)+2 \nu_{j} \omega_{s_{k}}+\nu_{j}^{2}\right] \stackrel{\text { def }}{=} \Delta_{1}+\Delta_{2}+\Delta_{3} .
$$

In view of (43), (51), and the definition of $h$,

$$
\Delta_{1} \leqslant \frac{a}{N} \sum_{j=1}^{n} \frac{1}{\widetilde{n}^{2}} \bigvee\left(C \tau \omega_{j}^{2}\right) \leqslant C\left(\tau+\tilde{n}^{-1}\right) \Psi .
$$

For any $j \in\left[s_{k}, r_{k}\right]$, we obtain $\nu_{j} \leqslant\left(\widehat{\gamma}_{j}-\gamma_{s_{k}}\right)_{+} /\left(\gamma_{s_{k}}+a / N\right)$ and $\widehat{\gamma}_{j}=$ $\min _{i \leqslant j} \widehat{\gamma}_{[i, \widehat{q}(j, n)]} \leqslant \widehat{\gamma}_{\left[s_{k}, \widehat{q}(j, n)\right]} \leqslant \gamma_{s_{k}}+\max _{i \geqslant j} \widehat{\gamma}_{\left[s_{k}, i\right]}$. From these inequalities and Corollary 3 , it follows that

$$
\frac{a}{N} \sum_{j=s_{k}}^{r_{k}} \mathbf{E} \nu_{j}^{2} \leqslant \sum_{j=s_{k}}^{r_{k}} C_{f} \lambda\left(j-s_{k}\right) \frac{N}{N_{1}^{2}} \leqslant C_{f} \ln ^{3} \tilde{n} \cdot \frac{N}{N_{1}^{2}} .
$$

Taking into account (43) and the inequality $h<C \ln \tilde{n} / \tau$, we find that

$$
\Delta_{3} \leqslant \frac{C \ln ^{4} \tilde{n}}{\tau M}\left(\frac{N}{N_{1}}\right)^{2} \Psi .
$$

Finally, (43), (51), and the Cauchy-Schwartz inequality lead to the estimate

$$
\Delta_{2} \leqslant 4\left(\sum_{j} \omega_{j}^{2} \frac{a}{N}\right)^{1 / 2} \Delta_{3}^{1 / 2} .
$$

Inequalities (52)-(56) demonstrate that (49) is valid for (50) as well, since $\widehat{\widehat{\omega}}_{j} \leqslant \widehat{\omega}_{j}, j \in \mathbf{N}$. Thus, (38) is proved.

Lemma 5. Let $\widehat{a}$ be given in (14), where $N_{0} \geqslant N^{-\alpha} / C$ for some fixed $\alpha \in\left(\frac{1}{2}, 1\right)$. There exists $\nu>0$ such that

$$
\mathbf{P}\left\{\left|\widehat{a}-a_{f}\right|>N^{-\nu}\right\} \leqslant C_{f} N^{-2} .
$$


P r o o f. We have $\widehat{a}=\left(\pi N_{0}\right)^{-1} \sum_{t \in \Pi_{0}} Z_{t}$, where $Z_{t}=\psi_{N}\left(X_{t}\right), t \in \Pi_{0}$. Obviously,

$$
0 \leqslant a_{f}-\mathbf{E} \widehat{a} \leqslant C_{f} \int_{0}^{N^{-1 /(2 q)}} \frac{d z}{\sqrt{z}} \leqslant C_{f} N^{-1 /(4 q)} .
$$

In view of the independence of $Z_{j}, j \in \Pi_{0}$, and the well-known relationships between moments and cumulants, one can write

$$
\begin{aligned}
\mathbf{E}(\widehat{a}-\mathbf{E} \widehat{a})^{2 k} & \leqslant C_{1}(k) \max _{2 \leqslant j \leqslant 2 k}\left|\operatorname{Cum}_{j}(\widehat{a})\right|^{2 k / j} \\
& =C_{1}(k) \max _{2 \leqslant j \leqslant 2 k}\left|\frac{1}{\pi^{j} N_{0}^{j-1}} \operatorname{Cum}_{j}\left(\widehat{Z}_{1}\right)\right|^{2 k / j} \\
& \leqslant C_{2}(k) \max _{2 \leqslant j \leqslant 2 k}\left(\mathbf{E}\left|\widehat{Z}_{1}\right|^{j} \cdot N_{0}^{1-j}\right)^{2 k / j},
\end{aligned}
$$

where $C_{1}(k), C_{2}(k)$ are constants dependent on $k$ only. Since

$$
\mathbf{E}\left|\widehat{Z}_{1}\right|^{j} \leqslant\|f\|_{\infty} N^{j / 2-1} \int_{-1}^{1} \psi_{N}(x) \mu(d x) \leqslant C_{f} N^{j / 2-1},
$$

inequality (57) follows from (58)-(60) and the Chebyshev inequality.

Let $\|V\|_{2}$ denote the Euclidean norm of a matrix $V=\left(v_{i j}\right)_{i, j=1}^{k}$ and let $|V|=\max _{i, j}\left|v_{i j}\right|$.

Lemma 6. Let $V$ be a symmetric matrix and its elements $v_{i j}=0$ for all $i, j$ provided $|i-j|>m$. Let $\Lambda=\operatorname{diag}\left\{\Lambda_{1}, \ldots, \Lambda_{k}\right\}$ be a diagonal matrix with the elements satisfying inequalities $0 \leqslant \Lambda_{1} \leqslant \cdots \leqslant \Lambda_{k} \leqslant 1$. Then for $k>m$,

$$
\left\|\Lambda V-\Lambda^{1 / 2} V \Lambda^{1 / 2}\right\|_{2}^{2} \leqslant\|V\|^{2} m(m+1) .
$$

P r o o f. According to the definition of $\Lambda$ we have

$$
\begin{aligned}
\left\|\Lambda V-\Lambda^{1 / 2} V \Lambda^{1 / 2}\right\|_{2}^{2} & =\sum_{i, j=1}^{k} v_{i j}^{2} \Lambda_{i}\left(\Lambda_{i}^{1 / 2}-\Lambda_{j}^{1 / 2}\right)^{2} \leqslant|V|^{2} \sum_{i=1}^{k} \sum_{|i-j| \leqslant m}\left(\Lambda_{i}-\Lambda_{j}\right)^{2} \\
& \leqslant|V|^{2} \sum_{i=1}^{k} \sum_{|i-j| \leqslant m}\left|\Lambda_{i}-\Lambda_{j}\right| .
\end{aligned}
$$

A simple algebra shows that

$$
\sum_{i=1}^{k} \sum_{|i-j| \leqslant m}\left|\Lambda_{i}-\Lambda_{j}\right|=2 \sum_{l=1}^{m} \sum_{r=1}^{l}\left(\Lambda_{k-l+r}-\Lambda_{r}\right) \leqslant m(m+1) .
$$

To complete the proof substitute this inequality into (62). 


\subsection{Proof of the main results.}

$\mathrm{Pr}$ o of of $\mathrm{Th}$ e o r e $\mathrm{m}$ 1. The analogous result (with a statistical estimate substituted for the functional $a_{g}$ in the definition of $f^{*}$ ) is established in [32, Theorem 4 and Example] under the additional restrictions on the sequence $b$. Lemma 6 shows that these restrictions are redundant. In fact, to make sure of this, it suffices to replace reference [32, (A.29)] by (61).

$\mathrm{Pr}$ o of of $\mathrm{Th}$ e o r e $\mathrm{m} 2$. Since we are considering the asymptotic behavior as $N \rightarrow \infty$, everywhere below we assume that $N$ is a sufficiently large number. Further $C$ denotes various constants dependent only on $C_{1}$ (see (1)). Let $f \in W_{g, b}, b \in B, a=a_{f}, M=M(b, N)$ (see (5)), and let $\Psi_{(\cdot)}$ be defined by (23). Set $\Psi_{n}=\min _{\omega \in \Omega} \Psi_{a, n}(\omega)$. The sequence $\Psi_{n}$ is nondecreasing and its limit $\Psi_{\infty}=\Delta(f, c) \leqslant \Delta(g, b)(1+o(1))$. Suppose that a natural number $m=m(N)$ is chosen so that $M=o(m)$. Then by virtue of (3) we obtain $b_{m}=o\left(b_{M+1}\right)=o(M / N)$. Since $\Psi_{m}-\Psi_{\infty} \leqslant b_{m}$,

$$
\Psi_{m} \leqslant \Delta(g, b)(1+o(1)), \quad \Delta(g, b) \asymp M N^{-1},
$$

by Theorem 1 . Here and everywhere below the convergence is uniform with respect to $f \in W_{g, b}$. The construction of the estimator

$$
f^{*}=u_{0}+\sum_{j=1}^{n^{*}} \omega_{j}^{*} c^{*}{ }_{j} u_{j}
$$

ensures the equality

$$
\delta\left(f, f^{*}\right)=\mathbf{E}\left[\Psi_{a, n^{*}}\left(\omega^{*}\right) \frac{N}{N_{3}}+\sum_{j=1}^{n^{*}}\left(\omega_{j}^{*}\right)^{2}\left(\frac{a}{N_{3}}-v_{j}\left(N_{3}\right)\right)\right] .
$$

Hence, due to Lemma 1,

$$
\delta\left(f, f^{*}\right) \leqslant \mathbf{E}\left[\Psi_{a, n^{*}}\left(\omega^{*}\right) N N_{3}^{-1}\right] \cdot(1+o(1))+o\left(M N^{-1}\right) .
$$

Note that

$$
\frac{N}{N_{3}} \leqslant C \wedge\left(1-N^{\alpha-1}-\frac{C}{k^{*}}\right) \stackrel{\text { def }}{=} \rho\left(k^{*}\right) .
$$

Let $\widetilde{\omega}$ be defined as $\omega^{*}$ with $\widehat{a}$ replaced by $a$. By virtue of Lemma 5 , from (64) and (65) we get

$$
\delta\left(f, f^{*}\right) \leqslant \mathbf{E}\left[\Psi_{a, n^{*}}(\widetilde{\omega}) \rho\left(k^{*}\right)\right] \cdot(1+o(1))+o\left(M N^{-1}\right) .
$$

Take $\widehat{\hat{\omega}}$ as in Lemma 4 with $n=n(k)=2^{k}, N_{1}=N_{1}(k)=\operatorname{Int}(N /(4 k))$. Inequalities (63) and (66) show that to conclude the proof it suffices to verify that

$$
\mathbf{E} \sum_{k=1}^{s} \Psi_{a, n}(\widehat{\widehat{\omega}}) \rho(k) \mathbf{P}\left\{k^{*}=k\right\} \leqslant \Psi_{m}(1+o(1))+o\left(\frac{M}{N}\right),
$$


where $s=\operatorname{Int}\left(\ln _{2} N\right)$. Since $M \rightarrow \infty, N / M \rightarrow \infty$, one can assume in addition that $m \leqslant(M \ln M) \wedge(N / 2)$. Lemma 4 implies that

$$
\mathbf{E} \Psi_{a, n}(\widehat{\widehat{\omega}}) \leqslant \Psi_{n}(1+o(1)) \leqslant\left(\Psi_{m}+\Gamma_{(n, m]}\right)(1+o(1)), \quad \text { if } n \leqslant M^{2},
$$

and

$$
\mathbf{E} \Psi_{a, n}(\widehat{\widehat{\omega}}) \leqslant C \Psi_{m} \ln ^{7} n, \quad \text { if } n>M^{2} .
$$

For $n>M^{2}$, one has $\Gamma_{[n / 2, n]}=o(\sqrt{n} / N)$. Therefore, applying notation $K_{1}=\left(\ln _{2} M^{2}, s\right] \cap \mathbf{N}, \widehat{\Gamma}_{k}=\Gamma_{\left[2^{k-1}, 2^{k}\right]}\left(D_{k}\right)$, the definition of $k^{*}$, Lemma 2 , and Chebyshev inequality, we obtain

$$
\begin{aligned}
\sum_{k \in K_{1}} \ln ^{7} n(k) \mathbf{P}\left\{k^{*}=k\right\} & \leqslant C \sum_{k \in K_{1}} k^{7} \mathbf{P}\left\{\widehat{\Gamma}_{k}>\frac{2^{k \beta-1}}{N}\right\} \\
& \leqslant C \sum_{k \in K_{1}} k^{9} 2^{k(1-2 \beta)}=o(1) .
\end{aligned}
$$

Set $r=\operatorname{Int}\left(\ln _{2} m+1\right)$. Now we will estimate

$$
\Phi=\sum_{k<r} \Gamma_{(n, m]} \mathbf{P}\left\{k^{*}=k\right\} .
$$

Fix $k<r$. If $\Gamma_{(n, m]}>2 r 2^{\beta r} / N$, then for some integer $j, k<j \leqslant r$,

$$
\Gamma_{j} \stackrel{\text { def }}{=} \Gamma_{\left[2^{j-1}, 2^{j}\right]} \geqslant \frac{\Gamma_{(n, m]}}{r} \geqslant 2 \cdot \frac{2^{\beta r}}{N} .
$$

In this case Lemma 2 and the definition of $k^{*}$ imply

$$
\begin{aligned}
\mathbf{P}\left\{k^{*}=k\right\} & \leqslant \mathbf{P}\left\{\widehat{\Gamma}_{j} \leqslant \frac{2^{j \beta}}{N}\right\} \leqslant \mathbf{P}\left\{-\widehat{\Gamma}_{j} \geqslant \frac{\Gamma_{j}}{2}\right\} \\
& \leqslant \frac{C\left[\Gamma_{j}\left|D_{j}\right|^{-1}+\left|D_{j}\right|^{-2}\right]}{\Gamma_{j}^{2}} \leqslant \frac{C j}{N \Gamma_{j}} \leqslant \frac{C r^{2}}{N \Gamma_{(n, m]}} .
\end{aligned}
$$

From the above mentioned and the equality $\sum_{k} \mathbf{P}\left\{k^{*}=k\right\}=1$, it follows that

$$
\Phi \leqslant \frac{C r^{3}}{N}+\frac{C m^{\beta} r}{N}=o\left(\frac{M}{N}\right) .
$$

Since $\rho(k)=1+o(1)$ as $k \rightarrow \infty$, to finish the proof it suffices to show that

$$
\Psi_{m} \sum_{k<l} \rho(k) \mathbf{P}\left\{k^{*}=k\right\}=o\left(\frac{M}{N}\right)
$$

for some $l=l(N) \rightarrow \infty$. Indeed, (68)-(75) imply (67).

If $\Psi_{m}=o(M / N)$, then (75) follows from the inequality $\rho(k) \leqslant C$. Let $\Psi_{m}>\tau M / N$ for some fixed $\tau>0$. For any $h<m$, one can write $\Psi_{m} \leqslant C h / N+\Gamma_{[h, m]}+\Gamma_{[m, \infty)}$ and $\Gamma_{[m, \infty)}=o(M / N)$. Thus, there exists $h=2^{l}, l \in \mathbf{N}$, such that $h>\tau M / C$ and $\Gamma_{l} \geqslant \tau M /(C N \ln M)$. Again, (15), 
Lemma 2, and Chebyshev inequality yield that $\mathbf{P}\left\{k^{*}<l\right\}=o(1)$. Since $\tau>0$ is arbitrary, we arrive at (75).

$\mathrm{Pr}$ o of of $\mathrm{T}$ h e o r e $\mathrm{m} 3$ repeats essentially the arguments in the proofs of Theorem 1 and Theorem 2. In [32, Theorem 4] the analog of Theorem 1 is proved for more general orthonormal bases satisfying conditions $\left[32\right.$, (A)-(D)] which are very similar to (U0)-(U2) and (18) for $b \in \mathscr{B}_{+}$ except condition (B). Condition (B) in [32] is used in the derivation of the upper bound for the minimax risk of $f^{*}$ and can be successfully replaced by condition (U1). This fact is apparent, in particular, from the proof of Theorem 2. The relaxation of (U2) as compared to [32, (C)] is stipulated by the application of Lemma 6 instead of [32, (A.29)].

The demonstration of the adaptivity of $f^{*}$ does not use any special property of the basis $u$ except the explicit form (17) of the functional $a_{g}$ for Jacobi polynomials given in (6). By making use of (U1) it easy to build up an estimator of $a_{g}$ with the desirable properties. The details are omitted.

\section{REFERENCES}

1. Barron A., Birgé L., Massart P. Risk bounds for model selection via penalization. Probab. Theory Related Fields, 1999, v. 113, № 3, p. 301-413.

2. Бенткус Р. Ю. Об асимптотике минимаксного среднеквадратического риска статистических оценок спектральной плотности в пространстве $L^{2}$. - Литов. матем. сб., 1985 , т. 25 , № 1, с. 23-42.

3. Бенткус Р. Ю., Казбарас А.Р. Об оптимальных статистических оценках плотности распределения. - Докл. АН СССР, 1981, т. 258, № 2, с. 265-268.

4. Bretagnolle J., Huber C. Estimation des densités: risque minimax. - Z . Wahrscheinlichkeitstheor. verw. Geb., 1979, v. 47, № 2, p. 119-137.

5. Donoho D.L. Asymptotic minimax risk for sup-norm loss: solution via optimal recovery. - Probab. Theory Related Fields, 1994, v. 99, № 2, p. 145-170.

6. Donoho D. L., Johnstone I. M. Ideal spatial adaptation via wavelet shrinkage. Biometrika, 1994, v. 81, № 3, p. 425-455.

7. Donoho D. L., Johnstone I. M. Adapting to unknown smoothness via wavelet shrinkage. - J. Amer. Statist. Assoc., 1995, v. 90, № 432, p. 1200-1224.

8. Donoho D. L., Johnstone I. M. Minimax estimation via wavelet shrinkage. - Ann. Statist., 1998, v. 26, № 3, p. 879-921.

9. Donoho D.L., Liu R. C., MacGibbon K.B. Minimax risk over hyperrectangles, and implications. - Ann. Statist., 1990, v. 18, № 3, p. 1416-1437.

10. Donoho D. L., Johnstone I. M., Kerkyacharian G., Picard D. Density estimation by wavelet thresholding. - Ann. Statist., 1996, v. 24, № 2, p. 508-539.

11. Ефроймович С. Ю. Непараметрическое оценивание плотности неизвестной гладкости. - Теория вероятн. и ее примен., 1985, т. 30, в. 3, с. 524-534.

12. Efromovich $S$. On nonparametric regression for i.i.d. observations in a general setting. - Ann. Statist., 1996, v. 24, № 3, p. 1126-1144.

13. Ефроймович С. Ю., Пинскер М. С. Оценивание квадратично-интегрируемой спектральной плотности по последовательности наблюдений. - Проблемы передачи информации, 1981 , т. 17, № 3, с. 50-68.

14. Ефроймович С.Ю., Пинскер М.С. Оценивание квадратично-интегрируемой плотности вероятности случайной величины. - Проблемы передачи информации, 1982, т. 18, № 3, с. 19-38.

15. Ефроймович С. Ю., Пинскер М. С. Самообучающийся алгоритм непараметрической фильтрации. - Автоматика и телемеханика, 1984, № 11, с. 58-65. 
16. ЕФроймович С. Ю., Пинскер М. С. Самонастраивающийся алгоритм минимакского непараметрического оценивания спектральной плотности. - Проблемы передачи информации, 1986 , т. 22 , № 3 , с. 62-76.

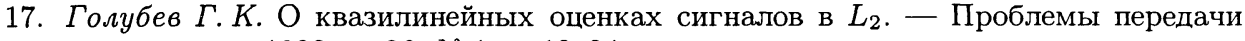
информации, 1990 , т. 26 , № 1, с. 19-24.

18. Голубев Г.К. Непараметрическое оценивание гладких плотностей распределения в $L_{2}$. - Проблемы передачи информации, 1992, т. 28 , № 1, с. 52-62.

19. Голубев Г. К. Непараметрическое оценивание гладких спектральных плотностей гауссовских стационарных последовательностей. - Теория вероятн. и ее примен., 1993 , т. 38 , в. 4 , с. $775-786$.

20. Golubev Yu.K., Levit B. Ya., Tsybakov A.B. Asymptotically efficient estimation of analytic functions in Gaussian noise. - Bernoulli, 1996, v. 2, № 2, p. 167-181.

21. Golubev G.K., Nussbaum M. A risk bound in Sobolev class regression. - Ann. Statist., 1990, v. 18, № 2, p. 758-778.

22. Голубев Г. К., Нуссбаум М. Адаптивные сплайновые оценки в непараметрической регрессивной модели. - Теория вероятн. и ее примен., 1992, т. 37, в. 3, с. 554-561.

23. Guerre E., Tsybakov A.B. Exact asymptotic minimax constants for the estimation of analytical functions in $L_{p}$. - Probab. Theory Related Fields, 1998, v. 112, № 1, p. 33-51.

24. Ибрагимов И. А., Хасьминский Р. З. Асимптотическая теория оценивания. М.: Наука, 1979, 528 c.

25. Kerkyacharian G., Picard D. Density estimation by kernel and wavelet method, optimality of Besov spaces. - Statist. Probab. Lett., 1993, v. 18, № 4, p. 327-336.

26. Коростелев А. П. Асимптотически минимаксная оценка регрессии в равномерной норме с точностью до постоянной. - Теория вероятн. и ее примен., 1993, т. 38, B. 4 , c. $875-882$.

27. Лепский О.В. Об одной задаче адаптивного оценивания в гауссовском белом шуме. - Теория вероятн. и ее примен., 1990 , т. 35, в. 3, с. 459-470.

28. Лепский О. В. Асимптотически минимаксное адаптивное оценивание. I. Верхние границы. Оптимально-адаптивные оценки. - Теория вероятн. и ее примен., 1991, т. 36 , в. 4 , c. $645-659$.

29. Лепский О.В. Асимптотически минимаксное адаптивное оценивание. II. Схемы без оптимальной адаптации. Адаптивные оценки. - Теория вероятн. и ее примен., 1992, т. 37 , в. 3, с. $468-481$.

30. Lepskii $O$. V. On problems of adaptive estimation in white Gaussian noise. - Topics in Nonparametric Estimation. Ed. by R. Z. Khasminskii. Providence, RI: Amer. Math. Soc., 1992, p. 87-106.

31. Пинскер М. С. Оптимальная фильтрация квадратично-интегрируемых сигналов на фоне гауссовского шума. - Проблемы передачи информации, 1980, т. 16, № 2 , c. $52-68$.

32. Radavičius $M$. Efficient nonparametric estimation of distribution density in the basis of algebraic polynomials. - Acta Appl. Math., 1995, v. 38, № 1, p. 13-35.

33. Radavičius $M$., Rudzkis $R$. Locally minimax efficiency of nonparametric density estimators for $\chi^{2}$-type losses. - Lithuanian Math. J., 1999, v. 39, p. 315-335.

34. Рудзкис $P$. Об одной оценке спектральной плотности. - Литов. матем. сб., 1985, т. 25 , № 3, c. 163-174.

35. Szegö G. Orthogonal Polynomials. Providence, RI: Amer. Math. Soc., 1959, 421 p.

36. Stone C. J. Optimal global rates of convergence for nonparametric regression. - Ann. Statist., 1982, v. 10, № 4, p. 1040-1053.

37. Цыбаков $A . Б$. Асимптотически эффективное оценивание сигнала в $L_{2}$ с общей функцией потерь. - Проблемы передачи информации, 1997, т. 33, № 1, с. 94-106.

38. Tsybakov A.B. Pointwise and sup-norm sharp adaptive estimation of functions on the Sobolev classes. - Ann. Statist., 1998, v. 26, №6, p. 2420-2469.

Поступила в редакцию

23.I. 2001

Исправленный вариант

28.V.2003 